\title{
Comportamiento que adoptan las parejas ante la infertilidad
}

\author{
William H. Onatra*; Elizabeth Acevedo C., Aurora Duque G., Hna. Lucila García G., Elsa Cristina Ramírez C.**
}

\begin{abstract}
RESUMEN: Se estudiaron 30 parejas de nivel socio-económico bajo que consultaron a la Clínica de Infertilidad del Instituto Materno Infantil de Bogotá durante el periodo enero/88 y febrero/89, con el fin de conocer el comportamiento adoptado ante el Diagnóstico de Infertilidad. Se encuentra que la relación de pareja es buena en el 33.3\% y regular/mala $23.3 \%$. Se presenta un "Cambio en la relación de pareja" en el $66.6 \%$, manifestado por enfriamiento de la relación conyugal $(\mathbf{5 8 . 8} \%)$ maltrato (17.6\%) y Adulterio (17.6\%). La expresión afectiva se modifica: Con disminución del cariño en el $29.7 \%$, convivir por obligación en el $24.3 \%$, insatisfacción sexual $21.6 \%$, despreocupación $18.9 \%$. La búsqueda de tratamiento se encuentra en el $60 \%$ y la conducta compensatoria inacción $40 \%$ búsqueda de empleo $23.3 \%$ cuidar niños ajenos $20 \%$ y deportes $10 \%$. La adopción como medida compensatoria es rechazada por los prejuicios que tienen sobre ésta. Se recomienda el trabajo de un equipo multidisciplinario de ginecólogos, enfermeras, psicólogos, terapeutas física y ocupacional, trabajadoras sociales como punto de apoyo para el manejo de las parejas que consultan por Infertilidad.
\end{abstract}

PALABRAS ClaVE: Infertilidad, Comportamiento Psicológico.

SUMMARY: 30 couples that consulted the Infertility Clinic of the Instituto Materno Infantil in Bogotá and belonging to to a low socio-economic level were studied during the period January 1988-february 1989 in order to evaluate the behaviour adopted in front of an infertilit diagnostic. The research shows that the couple relation is good in $33.3 \%$ and fair bad in $23.3 \%$. A change in the couple relation appears in $66.6 \%$, demonstrated in a cool conjugal relation, maltreat $(\mathbf{1 7 . 6} \%)$, adultery $(\mathbf{1 7 . 6} \%)$. The affective expression is modified by: love decrease $(29.7 \%)$, to live together by obligation $(24.3 \%)$, sexual insatisfaction $(21.6 \%)$, unprejudiced $(18.9 \%)$.

The searching of treatment is founded in a $60 \%$ and the compensation behaviour: inaction $40 \%$, searching of employment $23.3 \%$, taking care of anothers kids $20 \%$, sports $10 \%$. The adoption as a compensatory action is repeled, because they have prejudices.

It is reccomended the work of an multidisciplinary equipment: ginecologyst, urologyst, nurses, psicologysts physical and occupational therapeutics, social workers as a point of support for the management of the couples that consulted for infertility.

KEY WORDS: Infertility, psicology.

\section{Introducción}

Analizar el comportamiento que adopta la pareja que asiste a la consulta de infertilidad es un tema de gran interés, por los informes en la literatura sobre las diferentes reacciones que presentan las parejas ante la dificultad para concebir: dudas acerca de su masculinidad o feminidad, agresividad, aislamiento,disminución en la frecuencia del coito, depresión, angustia (1) (2). Esta situación hace que la pareja busque solución a éstos conflictos en diferentes sitios que le ofrezcan la posibilidad de un embarazo.

Para poder definir el comportamiento y la conducta humana revisamos algunos conceptos que diferentes investigadores tienen al respecto: Landauer y Pieron afirman que "el comportamiento es toda reacción o grupos de reacciones (respuestas) a un estímulo cualquiera"; Landauer dice que "la respuesta puede ser refleja o instintiva" y Pieron complementa su definición destacando "que el estímulo puede tener su origen tanto en el exterior como en el propio organismo" (3). Whittaker (4) añade a la definición anterior "Que el comportamiento se refleja en actos de un individuo, que

* Instructor Asociado. Departamento de Ginecología y Obstetricia. Universidad Nacional de Colombia. Instituto Materno Infantil. Bogotá.

** Estudiantes Décimo Semestre. Facultad de Enfermería. Universidad Javeriana. pueden ser observados objetivamente, registrados y estudiados, incluyendo los actos y los no verbales. Piaget, Watson y Skiner al referirse a la conducta están de acuerdo con lo que exponen los anteriores autores (3) (4) (5).

Un punto que vale la pena resaltar es la diferencia entre actitud y comportamiento. Morgan (6) afirma que "la actitud es una tendencia a responder positiva o negativamente ante ciertos objetos, personas o situaciones". Esto significa que las actitudes son predisposiciones para comportarse o responder ante estímulos. Ruck y Zimbardo dicen al respecto que "existen pruebas suficientes que aprueban la idea de que el cambio de actitudes se comprueba mejor desde la exposición a una situación en que se cambia de manera directa la conducta (7). Muchas veces podemos ver que una persona se comporta de una manera conraria a su actitud, el juego de roles o el consentimiento público forzan a la gente a cambiar de actitud y por ende su comportamiento.

De otra parte, el comportamiento está influenciado por los estímulos internos, externos y medio ambiente. En la conducta también influyen ciertas características individuales -inteligencia, personalidad, estado mental, emocionesy el grado de participación de los miembros del grupo al cual pertenecen. Fitcher (8) afirma que "la socialización es un proceso de influjo mutuo entre una persona y sus semejantes, proceso que resulta de aceptar las pautas de comportamiento social y de adaptarse a ellas". 
Por otra parte, las creencias y valores que determinan la conducta individual derivan en gran parte de la cultura y tienen que aprenderse. El proceso de aprendizaje está a cargo de los agentes de la cultura - padres, amigos, figuras de autoridad - y tienen lugar en todos los casos en forma de acontecimientos interpersonales.(9). Las personas crecen y se desarrollan en grupo social primario (familia) y reciben estímulos desde el nacimiento, las pautas establecidas permiten satisfacer las necesidades y determinan la conservación del grupo y del individuo. Consideramos de interés y por motivo de esta investigación conocer el comportamiento que presentan las parejas infértiles de bajo nivel cultural en las cuales el núcleo primario que moldearía su conducta no ha existido o está presente en forma inadecuada.

\section{Material y Métodos}

Se tomaron al azar 30 parejas de la Clínica de Infertilidad del Instituto Materno Infantil de Bogotá entre enero de 1988 y diciembre de 1989 , seleccionándose las que consultaron por Infertilidad Primaria. Se llevó a cabo una encuesta en la vivienda de cada una de las pacientes con el fin de evitar respuestas presionadas por el ambiente hospitalario.

Al elaborar la encuesta para precisar los términos de la misma se tuvieron en cuenta las siguientes definiciones:

Infertilidad Primaria: Incapacidad de concebir despúes de un año de relaciones sexuales regulares sin anticoncepción.

Pareja: Dos seres hombre y mujer que conforman una unidad conyugal con un buen equilibrio personal y entendimiento duradero.

Comportamiento: Según la definición de Skiner (4) "cualquier respuesta observable o medible de una persona o animal, que se produce ante un estímulo externo o interno". En nuestro estudio, comportamiento se definió como las respuestas dadas por las parejas en las cuales la mujer presenta Infertilidad Primaria, siendo este el estímulo para el comportamiento de pareja.

Entre los comportamientos consideramos los siguientes:

a) Cambio en la relación de pareja: Al verse frustrados sus deseos de tener hijos, los miembros de la pareja adoptan diferentes respuestas que influyen en la relación. De estas tuvimos en cuenta:

- Disolución de la Unión: Rompimiento del compromiso que existe entre el hombre y la mujer.

- Maltrato: Trato dado por uno de los cónyuges al otro, en forma tal que lo atropelle de palabra o de obra.

- Adulterio: Violación del contrato conyugal del hombre con una o varias mujeres, o viceversa.

- Enfriamiento de las relaciones conyugales: En ocasiones la disolución no llega a efectuarse, pero suele haber un cambio en la relación de pareja que se traduce en una vida de limitadas expresiones afectivas.

b) Adopción: Es una forma de sustituir el deseo frustado de no poder tener un hijo propio, obteniendo un hijo ajeno ya sea con los requisitos o formalidades que prescriben las leyes o sin ellas.

c) Inacción: No intentan llevar a cabo ninguna acción que pueda dar solución al problema.

d) Búsqueda incesante de tratamiento: La pareja ante este problema no se conforma y de común acuerdo se aferran a cualquier posibilidad de tener descendencia, lo que lleva a visitar a muchos facultativos, homeópatas, curanderos, etc., para recibir una variedad de tratamientos.

e) Otras conductas compensatorias: Las conductas compensatorias implican una variedad sustitutiva para una motivación frustrada. El fracaso o pérdida de la autoestimación, que impulsa al esfuerzo por recuperar lo perdido en otras áreas de la conducta como tipo de trabajo, cuidar hijos ajenos, deporte, etc.

Dentro de las variables que intervinieron se tuvo en cuenta: Edad, Factor Económico, Sociocultural, tipo de unión conyugal.

\section{Cuadro No. 1}

PAREJA

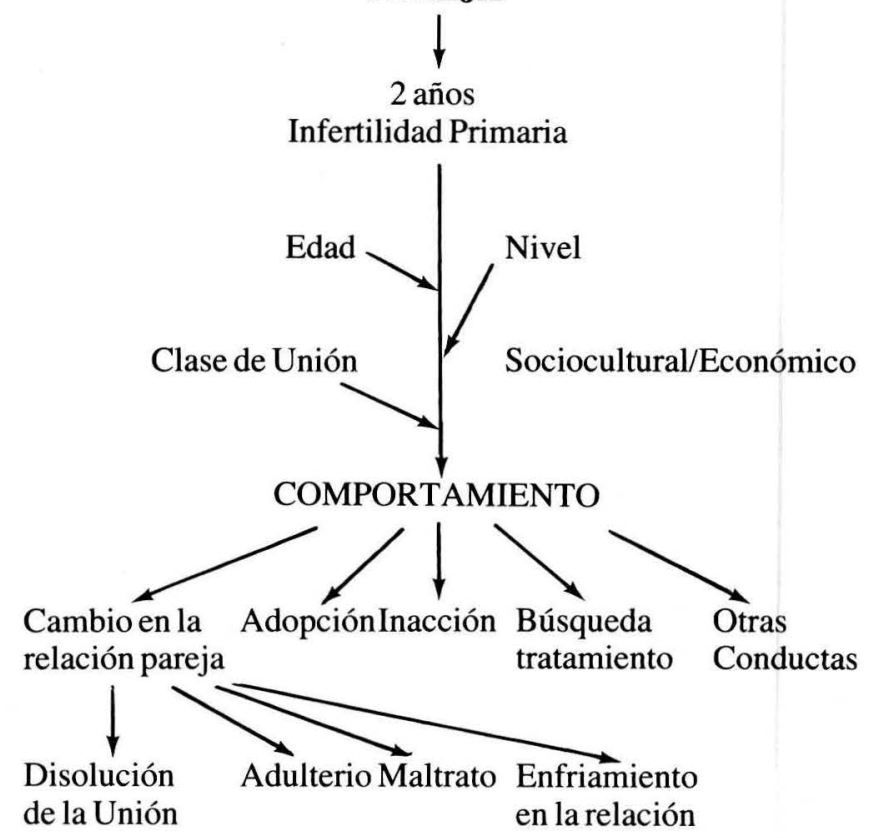

La tabulación de los datos de acuerdo con las respuestas se hizo en porcentaje, dada que la investigación no contempló la comparación con otra clase social.

\section{Resultados}

El rango de edad estuvo comprendido entre 18 y 35 años, con un promedio de 26.5. Solo $5(16 \%)$ tenían nivel Secundario y el resto apenas llegaban a cuatro de Primaria. La mayoría de las parejas 28 (93.3\%) estaban en Unión Libre y el $100 \%$ de ellas el nivel tanto socio-cultural como económico era Medio-bajo y Bajo.

A la pregunta de ¿cuál había sido el comportamiento de la pareja cuando se le informó sobre el diagnóstico de Infertilidad Primaria?. Como se puede apreciar en el Cuadro No. 1 , el cambio en la relación de pareja de "bueno a regular" y de "regular a malo" se encuentra en un $66 \%$ y el $33 \%$ no muestra modificación.

La distribución de cómo varía la relación de pareja Cuadro No. 2, Gráfica No. 2, demuestra que hay enfriamiento en las relaciones afectivas en el $58.8 \%$, con una proporción igual del $17.6 \%$, el adulterio y el maltrato. Llama la atención 
que no se encontró la separación como consecuencia de la Infertilidad.

Cuadro No. 1

DISTRIBUCION COMPARATIVA ENTRE LA RELACION DE LA PAREJA ANTERIOR Y POSTERIOR AL CONOCIMIENTO DEL DIAGNOSTICO DE INFERTILIDAD PRIMARIA EN LA MUJER

\begin{tabular}{|l|c|c|}
\hline COMPARACION DE & NUMERO DE & PORCENTAJE \\
LA RELACION & PAREJAS & \\
\hline Bueno - Buena & 10 & $33.33 \%$ \\
Bueno - Regular & 10 & $33.33 \%$ \\
Bueno - Mala & 1 & $3.33 \%$ \\
Regular - Malo & 7 & $23.33 \%$ \\
Regular - Buena & 2 & $6.66 \%$ \\
TOTAL & 30 & $100.00 \%$ \\
\hline
\end{tabular}

Cuadro No. 2

DISTRIBUCION DE LA FORMA EN QUE HA VARIADO

LA RELACION DE LAS PAREJAS EN LAS CUALES

LA MUJER PRESENTA INFERTILIDAD PRIMARIA

\begin{tabular}{|l|c|c|}
\hline $\begin{array}{l}\text { TIPO DE } \\
\text { VARIACION }\end{array}$ & $\begin{array}{c}\text { NUMERO DE } \\
\text { PAREJAS }\end{array}$ & PORCENTAJE \\
\hline Separoción & 0 & $0.00 \%$ \\
Adulterio & 6 & $17.64 \%$ \\
Maltrato & 6 & $17.64 \%$ \\
Enfriamiento en los rela- & & $58.82 \%$ \\
ciones ofectivas & 20 & $5.88 \%$ \\
\hline Otros & 2 & $100.00 \%$ \\
\hline
\end{tabular}

Gráfica No. 2

DISTRIBUCION DE LA FORMA EN QUE HA VARIADO

LA RELACION DE LAS PAREJAS EN LAS CUALES

LA MUJER PRESENTA INFERTILIDAD PRIMARIA

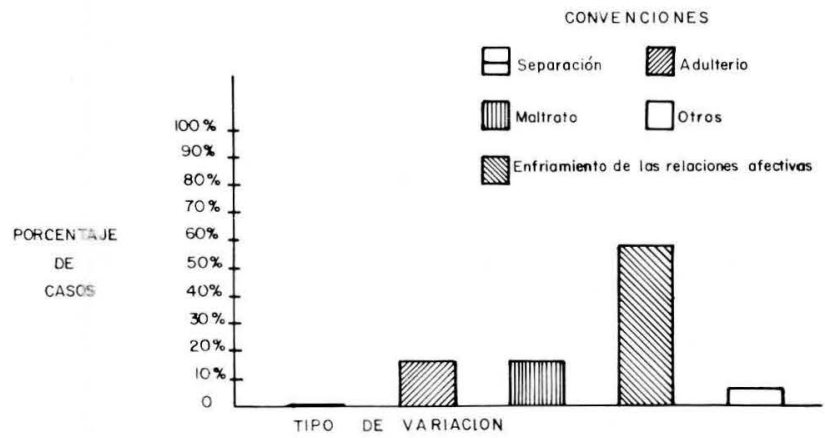

Respecto a la expresión afectiva de la pareja (Cuadro No. 3), encontramos que hay una disminución del cariño en el $(29.7 \%)$ y continuan viviendo por "obligación" en el $24.3 \%$. La disminución de la frecuencia de relaciones sexuales en el $21.6 \%$, la falta de preocupación $18.9 \%$ y más afecto $5.4 \%$ son algunas de las características de este grupo. En general podemos observar que hay una notable disminución de la afectividad en estas parejas.
Cuadro No. 3

DISTRIBUCION DE LA FORMA COMO OCURRE EL CAMBIO EN LA EXPRESION AFECTIVA DE LA PAREJA

$\left.\begin{array}{|c|c|c|}\hline \text { TI PO DE ENFRIAMIENTO } & \begin{array}{c}\text { NUMERO DE } \\ \text { PAREJAS }\end{array} & \text { PORCENTAJE } \\ \hline \text { DISMINUCION DEL CARINO } & 11 & 29.72 \% \\ \text { NO DESEO NI SATISFACCION DE LA } \\ \text { RELACION SEXUAL }\end{array}\right)$

El 73\% (22) de las parejas buscan atención médica para su problema y el $60 \%$ (18) de éstas persisten en el tratamiento. Cuadro No. 4. Estos datos demuestran que persiste una actitud de esperanza ante su infertilidad.

Cuadro No. 4

DISTRIBUCION DE LAS PAREJAS EN LAS CUALES LA MUJER PRESENTA INFERTILIDAD PRIMARIA Y CUYO TRATAMIENTO HA SIDO CONSTANTE

\begin{tabular}{|c|c|c|c|c|}
\hline \multirow{2}{*}{$\begin{array}{c}\text { BUSQUE DA DE } \\
\text { TRATAMIENTO }\end{array}$} & \multicolumn{2}{|c|}{$\begin{array}{c}\text { TRATAMIENTO } \\
\text { ACTUAL }\end{array}$} & \multicolumn{2}{c|}{ CONSTANCIA } \\
\cline { 2 - 5 } & Na. Parejas & Porcenta. & No. Parejas & Porcentaje \\
\hline SI & 22 & $73.33 \%$ & 18 & $60.00 \%$ \\
NO & 8 & $26.66 \%$ & 12 & $40.00 \%$ \\
TOTAL & $\frac{30}{100.00 \%}$ & $\frac{10}{30}$ & $\frac{100.00 \%}{}$ \\
\hline
\end{tabular}

Gráfica No. 5

DISTRIBUCION DE LAS PAREJAS EN LAS CUALES

LA MUJER PRESENTA INFERTILIDAD PRIMARIA Y QUE HAN ADOPTADO HIJOS

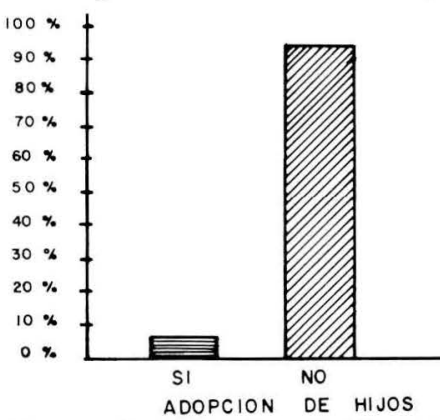

En relación con la Adopción Gráfica No. 5, únicamente $2(6 \%)$ habían adoptado un hijo, el resto del grupo mostró 
un claro rechazo ante esta posible solución a su infertilidad, $28(93.3 \%)$, aduciendo que se verían rechazados de su grupo social, el desconocer la procedencia de este hijo fueron algunos de sus argumentos.

Como conducta compensadora ante su infertilidad 12 casos $40 \%$ no toma ninguna actitud. Cuadro No. 6 Gráfica No. 6. Las restantes Intensifican el trabajo 7 (23.3\%) se dedican a cuidar niños ajenos $6(20 \%)$ hacen deportes 3 $(10 \%)$ y otros $2(6.6 \%)$. El comportamiento de inacción solo se presentó en 4 (13.3\%). Cuadro No. 7 .

Cuadro No. 6

DISTRIBUCION DE OTRAS CONDUCTAS COMPENSATORIAS ADOPTADAS POR LAS PAREJAS EN LAS CUALES LA MUJER PRESENTA INFERTILIDAD PRIMARIA

\begin{tabular}{|l|c|c|}
\hline $\begin{array}{l}\text { CONDUCTAS } \\
\text { COMPENSATORIAS }\end{array}$ & $\begin{array}{c}\text { NUMERO DE } \\
\text { CASOS }\end{array}$ & PORCENTAJE \\
\hline DEPORTES & 3 & $10.00 \%$ \\
CUIDAR NINOS AJENOS & 6 & $20.00 \%$ \\
NINGUNA & 12 & $40.00 \%$ \\
TRABAJAR MAS & 7 & $23.33 \%$ \\
OTROS & 2 & $6.66 \%$ \\
TOTAL & 30 & $100.00 \%$ \\
\hline
\end{tabular}

Cuadro No. 7

DISTRIBUCION DE LAS PAREJAS EN LAS CUALES

LA MUJER PRESENTA INFERTILIDAD PRIMARIA

Y QUE HAN ADOPTADO EL COMPORTAMIENTO DE INACCION

\begin{tabular}{|c|c|c|}
\hline INACCION & $\begin{array}{c}\text { NUMERO DE } \\
\text { PAREJAS }\end{array}$ & PORCENTAJE \\
\hline SI & 4 & $13.33 \%$ \\
NO & 26 & $86.67 \%$ \\
TOTAL & 30 & $100.00 \%$ \\
\hline
\end{tabular}

\section{Discusión}

Una vez tabulados los datos son varios los aspectos que esta encuesta nos demuestra. El nivel socio-económico bajo, con escolaridad primaria y el tipo de ocupación: obreros, pequeños comerciantes independientes, excepcionalmente con alguna profesión, es una muestra de la población que asiste al IMI. Estos oficios son generalmente mal remunerados, de contratos esporádicos no asegurando un salario fijo para la subsistencia de la familia, generando inestabilidad económica. Su vivienda localizada en los barrios sur-oriente, sur-occidente, en algunos casos, sin contar con los servicios sanitarios mínimos y en condiciones de hacinamiento. A pesar de esto llama la atención que estas parejas sin tener los recursos suficientes tratan de lograr un hijo explicable como postula Fischer (8): "el comportamiento adoptado por los individuos, influye en la socialización que es un proceso de influjo mutuo entre una persona y sus semejantes". Si tenemos en cuenta que en las familias de nivel socioeco- nómico bajo el promedio de hijos es de 6-10, las parejas con infertilidad en este estudio necesitan reafirmarse dentro del grupo social al cual pertenecen logrando una familia numerosa.

Respecto a la ocupación de las mujeres, se pudo determinar que el mayor porcentaje de ellas permanece en el hogar y esta soledad hace que se encierre más en su problema, las hace sentir improductivas no solo en el sentido de procreación sino también en lo económico. Al respecto, Whitaker (3) afirma, "los rasgos individuales son desarrollados de acuerdo con el propio status social en el que las personas basan el reconocimiento de su identidad y la posesión común de una serie de valores que se traducen en actitudes y patrones normalizados de comportamiento"; para estas mujeres en edades entre 18 - 35 años, etapa de la vida en la cual ya la persona debe tener definida su identidad, se presenta una crisis, al no poder cumplir el papel que le atribuye la sociedad -ser madre-, sino también como miembro activo de ella para contribuir con el ingreso familiar.

Se observó que el $66.6 \%$ de la muestra presentó "Cambio en la relación de pareja“" siendo generado principalmente por un enfriamiento gradual de las relaciones conyugales (58.2\%) manifestándose por una disminución del cariño, convivir por obligación, falta de preocupación, deterioro en las relaciones sexuales. Al plantearse la separación como alternativa ninguna de las parejas la acepta, algo paradójico dado el nivel cultural de este grupo. Al parecer la pareja desea mantener una apariencia a travéz de la cual logran la aprobación del grupo social. Hay que señalar que en dos parejas la relación varió, en el sentido de que el compañero se volvió más cariñoso y comprensivo, confirmando la hipótesis de Gutiérrez (10) "una de las maneras como la familia afronta los conflictos de la relación interconyugal, es comprender la situación".

Otros dos aspectos aunque se presentaron en un bajo porcentaje, pero que vale la pena mencionar son: El adulterio que se registró en un $17.64 \%$, principalmente, en el hombre lo cual es explicable si tenemos en cuenta que existe la creencia generalizada de que es la mujer la que tiene la incapacidad para concebir, haciendo que el hombre abandone el objetivo de lograr el hijo propio y como afirma Robinson (II) "el objetivo resulta muy importante para la persona". En la sociedad "machista" como en algunas áreas de Colombia, el número de hijos por fuera del hogar reafirma la virilidad de la persona. Hemos encontrado casos donde el hombre chantajea a la mujer si no le da un hijo. El otro aspecto es el Maltrato (17.64\%), el cual se puede relacionar con el hecho de que estas parejas conviven por obligación, en algunas ocasiones perdiéndose el respeto y la comprensión expresándose en agresión verbal y física. Este comportamiento es muy frecuente en clase socio-económica media y baja (12).

El otro comportamiento adoptado por las parejas fue la "Búsqueda incesante de tratamiento" $(60 \%)$, indicándonos que una vez diagnosticada la Infertilidad, la pareja no abandona su meta y busca por todos los medios a su alcance un tratamiento eficaz. Crombach (13) afirma, "cuando al individuo se le presenta un obstáculo para lograr un objetivo propuesto una de las maneras Para enfrentarse a ellas es la de no abandonar el objetivo y luchar hasta conseguirlo con 
un peligro grande si no lo consigue de generar frustración que puede llevar a un círculo vicioso y convertirse en una obsesión con el peligro de producir un transtorno mental en el individuo". Con base en esto el equipo que trabaja en esta área debe ser muy claro y honesto en los servicios que le puede prestar a la pareja y las limitaciones que se puedan presentar en cada caso con el fin de no dar esperanzas que finalmente desencadenen más frustración a la pareja.

A pesar de que hay parejas que no pierden la esperanza en lograr un embarazo el $60 \%$ adoptan "otras conductas compensatorias como una forma de adaptación a los fracasos encontrados. Al respecto diferentes autores (14) (15) (16), están de acuerdo con que "la compensación es interpretada como un concepto general que incluye múltiples formas específicas de adaptación a los fracasos e insuficiencias". En nuestro estudio encontramos que el $23.3 \%$ se ha dedicado a trabajar más porque la mujer trata de buscar la aprobación de su núcleo familiar y social aportando unos ingresos. El $20 \%$ decide cuidar niños ajenos, sustituyendo con esta acción la motivación intensa de desempeñarse en su rol como madre y reduciendo la tensión que le produce al no poderlo hacer con un hijo propio. Otras prefieren dedicarse a los deportes $\mathrm{u}$ otras actividades $(16.6 \%)$. Estas actitudes concuerdan con lo escrito por Allport (14) "la racionalización, la sublimación, el desplazamiento y otras formas de conducta, son técnicas compensatorias que hacen que el individuo afiance el funcionamiento de otro rasgo o característica capaz de apartar la atención de los demás de este defecto".

Las parejas que no adoptan ningún comportamiento, es decir, inacción (13.3\%) están de acuerdo con lo postulado por Crombach (13) "cuando el individuo se retira de su objetivo, elude su situación, pero no es por lo general una reacción deseable en los casos en que persiste la situación de fracaso", por lo tanto, el hecho de que la pareja no adopte ningún comportamiento ante el diagnóstico de Infertilidad puede ser una manifestación de esperanza, de éxito o una forma de expresión ante la frustración, creando a veces mayor tensión y angustia en la pareja. Algunas veces éstas parejas no adoptan ningún comportamiento porque no tienen los elementos suficientes para enfrentarse a esa situación haciéndose necesario algún tipo de orientación por parte del equipo encargado de atenderlas. (17)(18).

Por último, consideremos la adopción (6.6\%), como una situación clara de rechazo ante este tipo de conducta, explicable en parte por el nivel educativo de estas parejas, al proceso de socialización que genera perjuicios y desconfianza. Por otra parte, el hecho de llenar una serie de requisitos, demostrar solvencia económica, determinado nivel cultural y de personalidad, posibilidad de educar y ofrecerle un futuro a este hijo son limitantes para este grupo. (19) (20).

Por los hallazgos encontrados y dada la complejidad de los resultados encontrados desde el punto de vista psicológico se recomienda integrar un equipo multidisciplinario donde la parte médica brinde los diagnósticos y tratamientos adecuados a cada caso, con el apoyo de enfermería, psicología, terapia, trabajo social que seguramente brindarán un soporte afectivo a la angustia que estas parejas padecen.

\section{BIBLIOGRAFIA}

1. Seward GH, Wagner PS,Heinrich JF. The question of Psychophysiologic infertility: some negative answers. Psychososom Med 1965; 34:533.

2. Williams LS, Power PW. The emotional impact of infertility in single women. J Am Med Women Assoc 1977; 32:327.

3. Diccionario Terminológico de Ciencias Médicas. Sexta Edición. Edit. Salvat, Barcelona 1978.

4. Whittaker J. Psicología. Tercera Edición. Edit. Interamericana. México 1977.

5. Skinner W. El conductismo. Edit. Paidós. Barcelona 1976.

6. Cliffor M. Introducción a la Psicología. Cuarta Edición Edit. Aguilar. Madrid 1977.

7. Zimbardo P. Psicología de la Vida. Segunda Edición. Edit. Trillas, México. 1972.

8. Fichter J. Sociología. Tercera Edición. Edit. Helder. Barcelona 1974.

9. Lindgren H. Introducción a la Psicología Social. Primera Edición. Edit Trillas. México 1975.

10. Gutiérrez V. La Sociedad Colombiana. Cuarta Edición. Edit. Bedout. 1976.
11. Blejer J. Psicología de la Conducta. Tercera Edición. Edit. Universitaria. Buenos Aires. 1970.

i2. Fernández G. La Ciencia de la Conducta. Primera Edición. Edit. Trillas México. 1972.

13. Crombach H. Psicología . Tercera Edición. Editorial Norma Bogotá. 1975.

14. Allport G. La Personalidad. Edit. Herder. Barcelona 1973.

15. Brennar R.Psicología General. Primera Edition. Edit. Ariel Barcelona. 1975.

16. Díaz R. Hacia una teoría histórico-bio-psico-socio-cultural del comportamiento humano. Edit. Trillas. México. 1985.

17. Ardila R. La Psicología contemporánea. Segunda Edición. Edit. Paidós. Buenos Aires. 1971.

18. Climent C. Comportamiento y Salud Tercera Edición. Edit. Norma. Bogotá. 1975.

19. Ralf C. Anotaciones al estudio de las pacientes Infértiles. Primera Edición. Edit. Trillas. México. 1979.

20. Rosenfeld D,David L. Treating the emotional aspects of infertility: Counseling services in an Infertility Clinic. Am J. Obstet Gynecol 1979; 135:177. 